\title{
Hemoperitoneum Due to Penetrating Intercostal Artery Injury: A Case Report of a Rare and Still Understudied Entity
}

\author{
Maya Paran' ${ }^{1}$ Sivan Barkai ${ }^{1}$, Gerardo Camarillo ${ }^{1}$, Boris Kessel $^{1}$ \\ and Alexander Korin ${ }^{2}$ \\ 'Surgical Division, Hillel Yaffe Medical Center, Hadera, Israel, affliated with Rappaport Medical School, Technion, Haifa, Israel \\ 2Trauma Unit, Hillel Yaffe Medical Center, Hadera, Israel, affiliated with Rappaport Medical School, Technion, Haifa, Israel
}

\begin{abstract}
Intercostal artery injury may be life-threatening and usually presents as hemothorax. We report a unique case of penetrating injury, causing hemoperitoneum due to intercostal artery injury, without thoracic involvement. During urgent laparotomy, no intra-abdominal organ injury was found. Hemostasis was successfully achieved via suturing through an additional lateral $10 \mathrm{~cm}$ incision through the left thorax.
\end{abstract}

Keywords: Intercostal Artery; Penetrating Injury; Intraabdominal Injury

Received: 30 August 2021; Accepted: 30 September 2021

\section{INTRODUCTION}

Intercostal artery injuries have been reported to be caused by blunt and/or penetrating injuries [1] as well as iatrogenic injuries [2]. Traumatic injuries to the intercostal artery most commonly cause large hemothorax. We report a unique case of a penetrating injury, causing massive intra-abdominal bleeding from an intercostal artery, without any diaphragmatic injury or hemothorax.

\section{CASE PRESENTATION}

A 54-year-old male presented to a regional Level II trauma center after sustaining multiple stab wounds to his left chest at the posterior axillary line level, as well as the left back and both buttocks. On admission, the patient was noted to have active bleeding from his wounds, controlled by pressure dressings. Initial chest

\section{Corresponding author:}

Maya Paran MD, Hillel Yaffe Medical Center, Ha-Shalom St. Hadera, Israel.

Email: paran.maya@gmail.com

(C) 2021 CC BY 4.0 - in cooperation with Depts. of Cardiothoracic/ Vascular Surgery, General Surgery and Anesthesia, Örebro University Hospital and Örebro University, Sweden
$\mathrm{X}$-ray performed at the trauma bay was interpreted as normal. The patient remained hemodynamically stable and was referred to the radiology department for computed tomography (CT) scan of the chest and abdomen. Chest CT did not reveal any pathological findings, with no pneumohemothorax. Abdominal CT revealed a significant hemoperitoneum (Figure 1), with active extravasation from the left intercostal artery in the posterior aspect of the tenth intercostal space (Figure 2). The patient progressively developed hemodynamic instability and underwent emergency laparotomy. On surgery, approximately 2 liters of blood were evacuated from the abdominal cavity. Exploration of the abdomen did not reveal any parenchymal or hollow viscus injury. Active bleeding was detected in the posterior abdominal wall, which was treated with simple suturing followed by packing. However, this was not sufficient for control of bleeding. Therefore, it was decided to attempt control through an additional lateral incision. A $10 \mathrm{~cm}$ incision was performed through the stab wound on the lateral aspect of the left thorax, similar in location to a posterior lateral thoracotomy incision. Via this approach, hemostasis was achieved by sutures pulled through the chest wall from the inside out, around the ribs adjacent to the bleeding intercostal artery. During surgery, the patient received 4 units of packed red blood cells and 2 units of fresh frozen plasma. The patient was stabilized, underwent primary abdominal closure, and transferred 


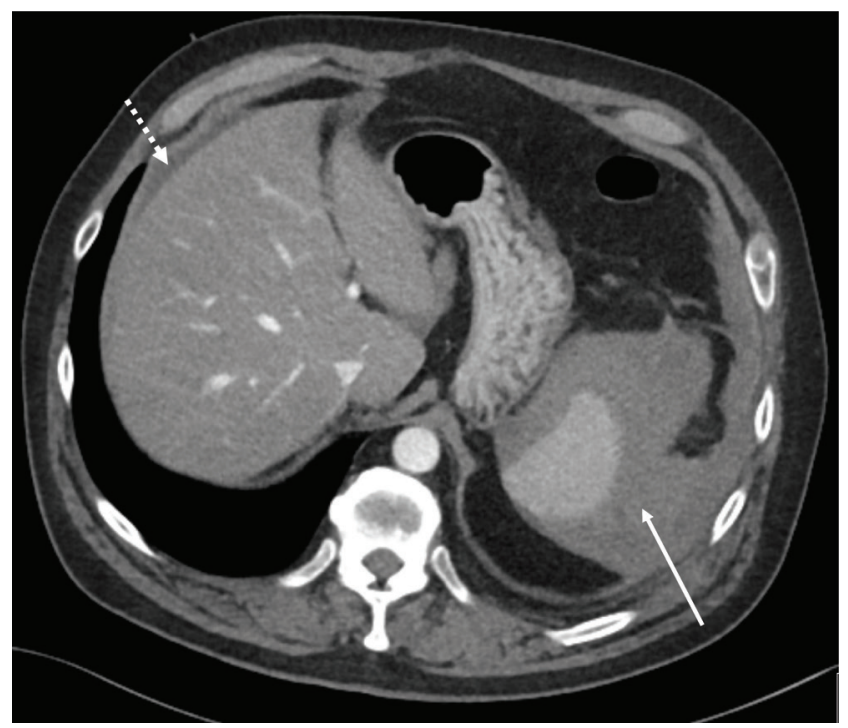

Figure 1 Abdominal CT showing significant hemoperitoneum. The white arrow shows hemoperitoneum around the spleen. The white shattered arrow shows blood in the right subdiaphragmatic space.

to the intensive care unit. The patient was weaned from mechanical ventilation the following morning. The postoperative course was without complications, hemoglobin levels remained stable, and the patient was uneventfully discharged on postoperative day 7 .

\section{Ethical Approval and Informed Consent}

Ethical approval was not required, informed consent was not required and all data was annonymized.

\section{DISCUSSION}

Intercostal artery injuries are occasional but potentially fatal injuries. The real incidence of such injuries either resulting from blunt or penetrating mechanisms remains unclear. Most studies on this topic include case series or single case reports alone. For example, during an eightyear period Tamburini et al. [3] described only 18 patients with traumatic intercostal artery injury. In this work, the mortality rate was $23 \%$ and the incidence of penetrating injury was not reported. In a different study, which collected data regarding both iatrogenic and blunt trauma patients with intercostal artery injury, Chemelli et al. [4] reported an overall mortality of $30 \%$ among 24 patients.

Hemorrhage control in such cases may be achieved surgically or by angioembolization [5]. The latter is only relevant in scenarios of hemodynamically stable patients. When approaching these arterial vessels intraoperatively, hemostasis can be achieved by suture ligation. However, in some cases surgical hemostasis can be very difficult. The literature clearly points out the challenge of controlling posterior intercostal bleeding,

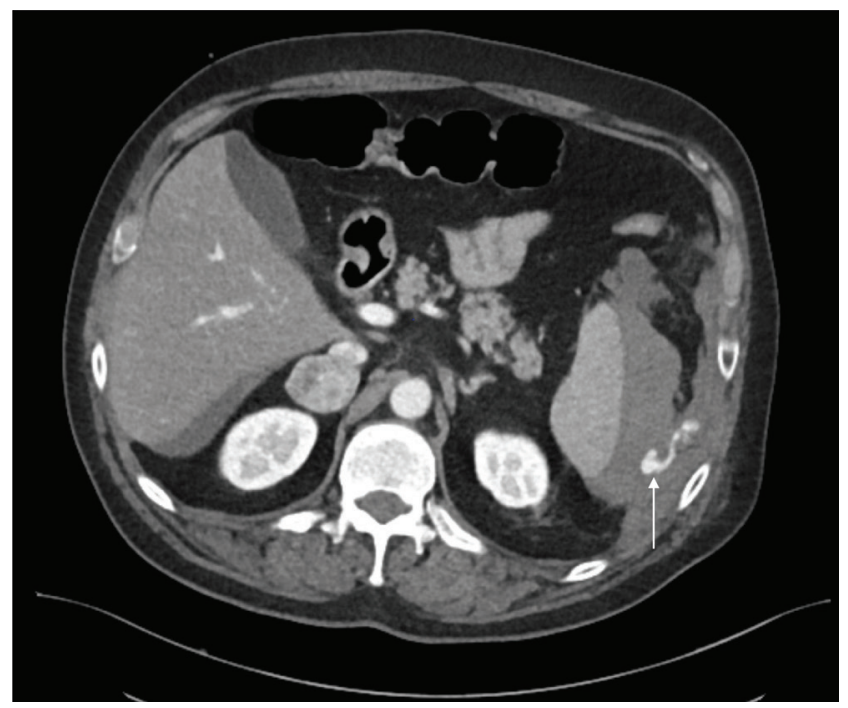

Figure 2 Abdominal CT showing active bleeding from the left intercostal artery. The white arrow shows active extravasation from the left intercostal artery in the posterior aspect of the tenth intercostal space.

mostly due to the limited space/exposure where the ribs have the least mobility and the intercostal space is smallest [6]. The accepted practice is performing a posterior lateral thoracotomy. However, previously described cases reported that the clinical manifestation of intercostal artery injury is mostly massive hemothorax. To the best of our knowledge, only a single case of hemoperitoneum necessitating laparotomy due to an intercostal artery injury, resulting from blunt chest trauma and rib fractures, has been reported [7]. This is the first description of an isolated massive hemoperitoneum caused by bleeding from an intercostal artery due to a penetrating injury. In our opinion, despite the findings on the CT scan, in the presence of a significant amount of blood in the abdominal cavity, the explorative laparotomy and meticulous revision of the abdominal cavity were mandatory in order to exclude intra-abdominal organ injury. In retrospect, a midline incision substantially restricted the exposure of the posterior intercostal space. As a result, additional posterior lateral thoracotomy was required to provide an adequate approach for hemostasis achievement. This created a unique problem, associated with placing a patient with an open and packed abdomen in a lateral decubitus position.

We believe that this case raises the possibility that some patients with a penetrating flank/back injury and massive hemoperitoneum may have an intercostal artery injury, even without hemothorax or diaphragmatic injury. A posterior wound may possibly raise a suspicion of an intercostal artery injury. This awareness is even more important in unstable patients who are operated upon immediately, without additional imaging. 


\section{Ethics Statement}

(1) All the authors mentioned in the manuscript have agreed to authorship, read and approved the manuscript, and given consent for submission and subsequent publication of the manuscript.

(2) The authors declare that they have read and abided by the JEVTM statement of ethical standards including rules of informed consent and ethical committee approval as stated in the article.

\section{Conflicts of Interest}

We confirm that there are no known conflicts of interest associated with this publication.

\section{Funding}

The authors received no financial support for the research, authorship, and/or publication of this article.

\section{REFERENCES}

[1] O'Connor JV, Byrne C, Scalea TM, Griffith BP, Neschis DG. Vascular injuries after blunt chest trauma: diagnosis and management. Scand J Trauma Resusc Emerg Med. 2009;17(42).

[2] Psallidas I, Helm EJ, Maskell NA, et al. Iatrogenic injury to the intercostal artery: aetiology, diagnosis and therapeutic intervention. Thorax. 2015;70: 802-4.

[3] Tamburini N, Carriel N, Cavallesco G, et al. Technical results, clinical efficacy and predictors of outcome of intercostal arteries embolization for hemothorax: a two-institutions' experience. J Thorac Dis. 2019;11(11): 4693-9.

[4] Chemelli AP, Thauerer M, Wiedermann F, Strasak A, Klocker J, Chemelli-Steingruber IE. Transcatheter arterial embolization for the management of iatrogenic and blunt traumatic intercostal artery injuries. J Vasc Surg. 2009;49(6):1505-13.

[5] Hagiwara A, Yanagawa Y, Kaneko N, et al. Indications for transcatheter arterial embolization in persistent hemothorax caused by blunt trauma. J Trauma. 2008;65(3):589-94.

[6] Hirshberg A, Mattox K. Top Knife: The Art \& Craft of Trauma Surgery. Castle Hill Barns, Harley, Nr Shrewsbury, UK: tfm Publishing Ltd; 2005. pp. 171-4.

[7] Laeeq K, Cheung S, Phillips B. Hemoperitoneum secondary to intercostal arterial bleeding in a trauma patient. J Surg Case Rep. 2017;2017(1):rjw194. 